Revista Colombiana de Obstetricia y Ginecología Vol. 57 No. 2 • $2006 \bullet(74-81)$

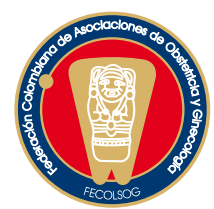

Investigación Original

\title{
FACTORES ASOCIADOS CON PARTO PRETÉRMINO EN EL TERCER NIVEL DE ATENCIÓN EN SALUD EN MANIZALES. JULIO 2004 - JUNIO 2005
}

Factors associated with preterm deliveries in third-level health centres in Manizales from Jully 2004 to Juine 2005

John Jairo Jaramillo-Prado, M.D. *, Irma Rocío López-Giraldo, M.D.**, Fernando Arango-Gómez, M.D.***

Recibido: marzo 15/06 - Revisado: abril 17/06 - Aceptado: mayo 16/06

\section{RESUMEN}

La incidencia del parto pretérmino se estima en un 10 a 11\%, siendo la principal causa de morbilidad y mortalidad perinatal sin que esto haya cambiado durante las últimas décadas.

Objetivo: identificar los factores asociados de las pacientes obstétricas con diagnóstico de parto pretérmino en el tercer nivel de atención de Manizales.

Metodología: se realizó un estudio de casos y controles en la Clínica Villapilar de la E.S.E. Rita Arango Álvarez del Pino que incluyó 232 pacientes que tuvieron el parto entre las semana 24 y 36 de gestación (casos) y 232 pacientes con parto después de la semana 37 (controles), durante el periodo de julio de 2004 a junio de 2005. En el análisis se determinó la asociación de ciertos factores de riesgo con el parto pretérmino mediante OR y sus respectivos intervalos de confianza al 95\%.

\footnotetext{
* Gineco-Obstetra, Universidad de Caldas, Manizales.

Correo electrónico: johnjamedico@hotmail.com

Dirección: carrera 22 \# 46 ${ }^{\mathrm{a}}$-64 apto 202.

** Gineco-Obstetra, Instructora Asociada, Universidad de Manizales.

** Pediatra, Neonatólogo, Profesor Asistente, Universidad de Caldas.
}

Resultados: la no realización de control prenatal (OR 5,1 IC95\% 2,41-11,01), preeclampsia durante el embarazo actual (OR 4,15 IC95\% 1,70-10,16), ruptura prematura de membranas (OR 3,17 IC95\% 1,92-5,23), embarazo múltiple (OR 7,65 IC95\% 2,77-21,16) y el antecedente de parto prematuro (OR 5,59 IC95\% 2,46-12,73) tuvieron asociación estadísticamente significativa con la presentación de parto prematuro. No hubo diferencias en las variables de edad, estado civil, procedencia, estado socio-económico, gravidez, paridad, planificación familiar, enfermedades de transmisión sexual y hábitos nocivos entre los grupos de casos y controles.

Conclusiones: los principales factores asociados a parto pretérmino en el tercer nivel de atención en Manizales fueron el antecedente de parto pretérmino, control prenatal inadecuado y la asociación de la gestación actual con ruptura prematura de membranas, preeclampsia y/o gestación múltiple.

Palabras clave: parto pretérmino, factores de riesgo, estudios de casos y controles. 


\section{SUMMARY}

The incidence of preterm delivery is estimated to be $10 \%$ to $11 \%$ of all pregnancies, this being the main cause of morbidity and perinatal mortality which has remained unchanged during the past few decades.

Objective: identifying factors associated with obstetric patients diagnosed as suffering from preterm delivery in third-level attention centres in Manizales.

Methods: a clinical study of cases and controls was carried out in the Villapilar ESE Rita Arango Alvarez del Pino clinic. This included 232 patients with 24- to 36-week delivery (cases) and 232 patients with 37-week delivery or longer (controls) from July 2004 to June 2005. Risk factors associated with preterm delivery were determined by OR and $95 \%$ confidence interval.

Results: there was statistical association between non-prenatal care (OR 5.1 IC95\% 2.41-11.01), preeclampsia during pregnancy (OR 4.15 IC95\% 1.70-10.16), premature rupture of membranes (OR 3.17 IC95\% 1.92-5.23), multiple gestation (OR 7.65 IC95\% 2.77-21.16) and previous preterm delivery (OR 5.59 IC95\% 2.46-12.73).

There was no significant statistical difference regarding age, marital status, origin, socio-economical status, number of pregnancies, family planning, sexually transmitted diseases and harmful habits among the groups of cases and controls.

Conclusions: the main factors associated with preterm delivery in third-level attention centres in Manizales were previous preterm delivery, inadequate prenatal care and the association of premature rupture of membranes, preeclampsia and multiple gestation.

Key words: preterm delivery, risk factor, casecontrol studies.

\section{INTRODUCCIÓN}

El parto pretérmino es uno de los mayores problemas en obstetricia y ginecología con una incidencia aproximada de $10-11 \%{ }^{1}$ variando entre las diferentes poblaciones según los factores de riesgo que estén presentes; es la principal causa de morbilidad y mortalidad perinatal a nivel mundial, responsable del 70\% de las muertes neonatales ${ }^{2}$ y del $50 \%$ de las secuelas neurológicas en los nacidos menores de 32 semanas de gestación ${ }^{3}$ sin que esto haya cambiado en las últimas décadas.

Algunos estudios han intentado identificar los factores que incrementan el riesgo para parto pretérmino dentro de los cuales se han mencionado la historia de un parto prematuro previo, la edad materna, el control prenatal inadecuado y la raza negra entre otros. ${ }^{4}$ En nuestro país existen pocos estudios que destacan algunas condiciones predominantes en las pacientes con parto pretérmino.,

El objetivo de este trabajo es identificar los factores asociados de las pacientes obstétricas con diagnóstico de parto pretérmino que son remitidas para atención de tercer nivel en Manizales a un centro universitario, sitio de referencia principal del departamento de Caldas.

\section{MATERIALES Y MÉTODOS}

Se realizó un estudio de casos y controles en el tercer nivel de atención en salud en Manizales, tomando como población de estudio las gestantes que tuvieron su parto en la Clínica Villapilar de la E.S.E. Rita Arango Álvarez del Pino, institución de carácter universitario y principal centro de referencia del Departamento de Caldas, durante el periodo comprendido entre julio de 2004 y junio de 2005. Se definieron como casos a las madres que tuvieron su parto después la semana 24 y antes de la semana 37 de gestación, tanto por trabajo de parto pretérmino espontáneo, como inducido. Se definió control a las madres que tuvieron su parto después de las 37 semanas de gestación, los cuales fueron seleccionados tomando el siguiente nacimiento a término después de un parto pretérmino, en la misma institución. No se consideraron criterios de exclusión. 
El cálculo de la edad gestacional se realizó con base en la fecha de la última regla confiable o por ecografía antes de la semana 20. Cuando esta información no estaba disponible en la historia clínica, se determinó por examen físico del recién nacido mediante el método de Ballard modificado. ${ }^{7}$

La recolección de la información se realizó por parte de 4 médicas internas que hacían su práctica en el servicio de obstetricia y quienes no conocían los objetivos del estudio. Se aplicó un cuestionario con 28 variables que agrupaban factores demográficos, socioculturales, antecedentes ginecoobstétricos y patológicos. La información se obtuvo mediante entrevista directa a la paciente y posteriormente los datos se corroboraron por medio del análisis de la historia clínica actual, y cuando fue posible, con la historia clínica de consultas u hospitalizaciones anteriores. El estudio contó con la aprobación del comité de ética de la Universidad de Caldas y del Departamento Materno Infantil de la institución.

Se calculó un tamaño de muestra de sujetos con base en un nivel de significancia del 95\%, poder del $80 \%$, relación de casos: controles de 1:1, con una frecuencia esperada de trabajo de parto pretérmino en la población general del 7\% y una frecuencia esperada de trabajo de parto pretérmino en las madres que han tenido el antecedente de parto pretérmino en gestaciones anteriores del $16 \%{ }^{8}$

En el análisis se determinó la asociación de los factores de riesgo con el parto pretérmino mediante OR y sus respectivos intervalos de confianza al 95\%. Para la comparación de las variables sociodemográficas y obstétricas en los casos y los controles se empleó la prueba de ji cuadrado $\left(\chi^{2}\right)$ y se determinó un nivel de significancia del 95\%. El análisis se realizó con el programa Epiinfo ${ }^{\circledR}$ 2000, versión 3.32.

\section{RESULTADOS}

Se analizaron en total 464 pacientes atendidas en una institución de tercer nivel. El promedio de edad en el grupo de casos fue de 24,8 $\pm 7,3$, mientras que en el grupo control fue de 24,3 \pm 6,7 años.
No se encontraron diferencias en los antecedentes de paridad, abortos previos, inicio de relaciones sexuales, métodos de planificación familiar, número de compañeros sexuales ni hábitos nocivos (cigarrillo, alcohol y drogas). El antecedente de enfermedades de transmisión sexual fue de 12,1\% y 7,3\% respectivamente para los casos y controles, sin mostrar diferencias para ambos grupos. Las características de la población se resumen en la tabla 1 .

El número de controles prenatales fue clasificado en tres categorías (4, 1 a 3 y ninguno). En la tabla 2 se aprecia que la realización de control prenatal inadecuado fue mayor en el grupo de madres con parto pretérmino comparado con los controles. Las enfermedades concomitantes durante la gestación actual (trastornos hipertensivos del embarazo, infección de vías urinarias, vaginosis bacteriana y diabetes), se presentaron en el 58,2\% de los casos y $39,6 \%$ de los controles $(\mathrm{p}<0,05)$; el análisis individual sólo mostró diferencias significativas en cuanto a la preeclampsia. El análisis de condiciones obstétricas asociadas al embarazo actual como la ruptura prematura de membranas y el embarazo múltiple mostraron diferencias significativas para el grupo de madres con parto pretérmino. El antecedente de parto prematuro en embarazos anteriores fue mayor en el grupo de casos (tabla 3).

\section{DISCUSIÓN}

En estudios reportados previamente en la literatura se han encontrado asociaciones entre el riesgo aumentado de parto pretérmino en los extremos de la edad materna ( $<20$ y $>35$ años). ${ }^{4,9,10}$ Recientemente, Amaya y colaboradores ${ }^{6}$ demostraron asociación entre prematurez y edad materna menor de 15 años (RR 1,88, IC 95\% 1,14-3,11) en nuestro país. Nosotros no encontramos dicha asociación debido al pequeño número de pacientes en este grupo de edad (3 casos y 1 control). El bajo nivel socioeconómico y educativo han sido reportados como factores de riesgo para prematurez. ${ }^{10-12}$ En este estudio no encontramos diferencias en el estrato socio- 


\section{Tabla 1. Características sociodemográficas de los grupos.}

\begin{tabular}{|c|c|c|c|c|c|c|}
\hline & & Parto & $\operatorname{mino} *$ & Par & mino & Valor de $p$ \\
\hline & & $\mathbf{n}$ & $\%$ & $\mathbf{n}$ & $\%$ & \\
\hline & $<20$ & 67 & 28,9 & 65 & 28 & \\
\hline Edad (años) & $20-34$ & 134 & 57,7 & 140 & 60,4 & 0,8035 \\
\hline & $\geq 35$ & 31 & 13,4 & 27 & 11,6 & \\
\hline & Soltera & 68 & 29,3 & 64 & 27,6 & \\
\hline Fot a il & Casada & 59 & 25,4 & 49 & 21,1 & \\
\hline & Unión libre & 105 & 45,3 & 116 & 50 & \\
\hline & Otro & 0 & 0 & 3 & 1,3 & \\
\hline & Urbana & 162 & 69,8 & 166 & 71,6 & \\
\hline & Rural & 70 & 30,2 & 66 & 28,4 & \\
\hline & $1-2$ & 159 & 68,8 & 152 & 65,5 & \\
\hline $\begin{array}{l}\text { Estrato } \\
\text { socioeconómico }\end{array}$ & $3-4$ & 69 & 29,9 & 80 & 34,5 & 0,1376 \\
\hline & $5-6$ & 3 & 1,3 & 0 & 0 & \\
\hline & Ninguna & 11 & 4,7 & 9 & 3,9 & \\
\hline For lold & Primaria & 89 & 38,4 & 68 & 29,3 & \\
\hline & Secundaria & 121 & 52,2 & 146 & 62,9 & \\
\hline & Universidad & 11 & 4,7 & 9 & 3,9 & \\
\hline & No asegurada & 138 & 59,5 & 133 & 57,3 & \\
\hline $\begin{array}{l}\text { Seguridad } \\
\text { social** }\end{array}$ & Subsidiado & 52 & 22,4 & 41 & 17,7 & 0,1385 \\
\hline & Contributivo & 42 & 18,1 & 58 & 25 & \\
\hline & 1 & 100 & 43,1 & 104 & 44,8 & \\
\hline Gravidez & $2-3$ & 90 & 38,8 & 93 & 40,1 & 0,6825 \\
\hline & $\geq 4$ & 42 & 18,1 & 35 & 15,1 & \\
\hline
\end{tabular}

* No se encontraron diferencias significativas en ninguna de las variables $(\mathrm{p}>0,05)$, utilizando la prueba de ji cuadrado $\chi^{2}$ ).

** En Colombia, el Sistema General de Seguridad Social en Salud incluye dos regímenes, el contributivo, conformado por la población trabajadora con capacidad de pago, y el subsidiado constituido por la población pobre y vulnerable, sin capacidad de aportar económicamente al sistema. 
Tabla 2. Distribución de casos y controles según el número de controles prenatales.*

\begin{tabular}{|c|c|c|c|}
\hline $\begin{array}{c}\text { Controles } \\
\text { prenatales }\end{array}$ & Casos & Controles & $\begin{array}{c}\text { OR } \\
\text { (IC 95\%) }\end{array}$ \\
\hline $\mathbf{2} 4$ & 130 & 187 & 1,0 \\
\hline $\mathbf{1 - 3}$ & 63 & 34 & $\begin{array}{c}2,67 \\
(1,76-4,07)\end{array}$ \\
\hline Ninguno & 39 & 11 & $\begin{array}{c}5,1 \\
(2,41-11,01)\end{array}$ \\
\hline
\end{tabular}

* Se tomó como referencia las madres que asistieron a 4 o más controles prenatales y se compara el riesgo de prematurez con las madres que asistieron a 1 a 3 controles y con las madres que no asistieron a control.

económico, tipo de seguridad social y nivel educativo, lo cual refleja las características de la población que accede a esta institución de carácter público. Aunque la raza ha sido asociada por Goldenberg ${ }^{13,14}$ como una variable independiente que aumenta el riesgo de parto pretérmino, se decidió no incluirla entre las variables a estudio dado el gran número de mezclas de nuestra población y lo subjetivo que sería su evaluación.

Los comportamientos nocivos como el hábito de fumar, la dependencia al alcohol y el consumo de cocaína se han encontrado asociados significativa- mente con parto pretérmino en varios estudios. ${ }^{15-20}$ Sin embargo, Miller JM y colaboradores ${ }^{21}$ no han encontrado esta misma asociación en cuanto al consumo de cocaína se refiere. En el presente trabajo, aunque existió una tendencia mayor en los comportamientos nocivos en el grupo de los casos (10,3\% vs 6,5\%), ésta no fue significativa posiblemente debido al escaso número de pacientes que afirmaron tener dichos hábitos. Además, el abuso de estas sustancias ha sido asociado de forma más estrecha a la restricción del crecimiento fetal y los programas para disminuir su consumo han tenido potencial limitado para reducir la tasa global de parto pretérmino..$^{13,22}$

Encontramos una asociación entre las pacientes con parto pretérmino y control prenatal inadecuado, similar a lo reportado en otros estudios de tipo observacional; ${ }^{23,24}$ sin embargo, algunos estudios controlados de intervención han mostrado resultados desalentadores, ${ }^{25,26}$ lo cual hace que las intervenciones de control prenatal necesarias para lograr un impacto real permanezcan aún poco claras. En países en desarrollo como el nuestro, el impacto del control prenatal en la prevención de la prematurez puede ser mayor. ${ }^{27}$

El antecedente de parto prematuro ha sido descrito por Mercer y otros autores como el principal

\begin{tabular}{|c|c|c|c|c|}
\hline Condiciones asociadas & $\begin{array}{c}\text { Casos } \\
(\mathrm{n}: 232)\end{array}$ & $\begin{array}{l}\text { Controles } \\
\text { (n: 232) }\end{array}$ & $\begin{array}{l}\text { OR crudo } \\
\text { (IC 95\%) }\end{array}$ & $\begin{array}{l}\text { OR ajustado } \\
\text { (IC 95\%) * }\end{array}$ \\
\hline $\begin{array}{l}\text { Ruptura prematura } \\
\text { de membranas }\end{array}$ & $74 / 232$ & $28 / 232$ & $\begin{array}{c}3,41 \\
(2,05-5,69)\end{array}$ & $\begin{array}{c}3,17 \\
(1,92-5,23)\end{array}$ \\
\hline Preeclampsia & $57 / 232$ & $26 / 232$ & $\begin{array}{c}2,58 \\
(1,51-4,42)\end{array}$ & $\begin{array}{c}4,15 \\
(1,70-10,16)\end{array}$ \\
\hline Embarazo múltiple & $28 / 232$ & $5 / 232$ & $\begin{array}{c}6,23 \\
(2,24-18,75)\end{array}$ & $\begin{array}{c}7,65 \\
(2,77-21,16)\end{array}$ \\
\hline $\begin{array}{c}\text { Antecedente de parto } \\
\text { prematuro }\end{array}$ & $37 / 232$ & $8 / 232$ & $\begin{array}{c}5,31 \\
(2,31-12,69)\end{array}$ & $\begin{array}{c}5,59 \\
(2,46-12,73)\end{array}$ \\
\hline
\end{tabular}


factor de riesgo en multíparas, ${ }^{28-30}$ con un incremento de 2,5 veces el riesgo, ${ }^{7}$ lo cual se correlaciona claramente con la asociación encontrada en nuestra investigación (OR 5,31, IC 95\% 2,31-12,69). Lo anterior debe motivar la captación de estas pacientes siempre dentro de los controles de alto riesgo obstétrico tendiente a minimizar los factores de riesgo modificables que puedan estar presentes y a brindar la mayor calidad de atención posible.

La preeclampsia se ha asociado con parto prematuro, ${ }^{31,32}$ principalmente si se desarrolla antes de la semana 33 de gestación. ${ }^{33,34}$ En las pacientes con parto pretérmino, encontramos dicha complicación en $24,6 \%$, comparado con $11,2 \%$ en las pacientes pertenecientes al grupo control (OR 2,58 IC 95\% 1,51-4,42). De igual manera, la gestación múltiple se asocia con parto pretérmino. ${ }^{17,35,36} \mathrm{El} 57 \%$ de los embarazos gemelares y el $92 \%$ de los embarazos triples terminan en nacimientos prematuros. ${ }^{37} \mathrm{En}$ nuestro análisis se observó esta asociación (OR 6,21 IC 95\% 2,31-20,99). La ruptura prematura de membranas y su asociación al parto pretérmino fue un hallazgo consistente entre este estudio y la literatura mundial. Esta patología es responsable de una cuarta parte de los nacimientos prematuros en el mundo ${ }^{38}$ y continúa siendo un desafío para quienes a diario se enfrentan con esta entidad.

Este estudio tiene como limitaciones la utilización de un solo centro de referencia como fuente de las pacientes, lo cual limita las conclusiones a gestantes de características socioeconómicas similares a las del estudio y en centros de referencia del nivel III de atención. Existe un posible sesgo de selección diferencial al haber incluido tanto pacientes con parto pretérmino espontáneo como pacientes con parto pretérmino inducido, situación en la cual el factor de exposición es el que determina ser caso y no ser control. Posiblemente exista además, sesgo de información en el antecedente de enfermedad de transmisión sexual y el antecedente de parto pretérmino porque no siempre fue posible corroborar dicha información en las historias clínicas de consultas u hospitalizaciones anteriores. Además no introdujo algunas variables que han sido descritas como factores de riesgo de forma no consistente en otros estudios como son el estado nutricional de las pacientes, la anemia materna, el trabajo extremo y el estrés personal, dado que estas variables se encontraron inviables de recolectar de forma objetiva en todas las pacientes.

\section{CONCLUSIONES}

Los principales factores asociados a parto pretérmino en el tercer nivel de atención en Manizales fueron el antecedente de parto pretérmino, control prenatal inadecuado y la asociación de la gestación actual con ruptura prematura de membranas, preeclampsia y/o gestación múltiple lo cual está en acuerdo con lo reportado en otras poblaciones y debe dirigir la atención de los especialistas en el área para motivar la realización de estudios de intervención sobre estos factores encontrados y que permitan valorar su impacto en pacientes con características propias de nuestro país, las cuales distan de las encontradas en los países desarrollados.

\section{AGRADECIMIENTOS}

A las doctoras Paula Andrea López, Natalia Tamayo, Jimena Aristizábal, Lina Baños y profesores del Departamento Materno Infantil de la Universidad de Caldas por sus aportes a este trabajo.

\section{REFERENCIAS}

1. Creasy RK, Iams JD. Preterm labor and delivery. En: Creasy RK, Resnik R (eds). Maternal-Fetal Medicine. 4th ed. Philadelphia: WB Saunders Co; 1999. p. 498-531.

2. Pschirrer ER, Monga M. Risk factors for preterm labor. Clin Obstet Gynecol 2000;43:727-34.

3. Goldenberg RL. The management of preterm labor. Obstet Gynecol 2002;100:1020-37.

4. Iams JD. Preterm birth. En: Gabbe SG, Niebyl JF, Simpson JL (eds). Obstetrics: Normal and Problem Pregnancies. 3rd ed. New York: Churchill Livingstone; 1996. p. 743-820. 
5. Orozco J, Acosta G, Rojano A, Rhenals G. Parto prematuro y factores de riesgo asociados en el Hospital Universitario Metropolitano. Rev Colomb Obstet Ginecol 1995;46:179-85.

6. Amaya J, Borrero C, Ucrós S. Estudio analítico del resultado del embarazo en adolescentes y mujeres de 20 a 29 años en Bogotá. Rev Colomb Obstet Ginecol 2005;56:216-24.

7. Ballard JL, Khoury JC, Wedig K, Wang L, EilersWalsman BL, Lipp R. New Ballard Score, expanded to include extremely premature infants. J Pediatr 1991;119:417-23.

8. Mercer BM, Goldenberg RL, Moawad AH, Meis PJ, Iams JD, Das AF, et al. The preterm prediction study: effect of gestational age and cause of preterm birth on subsequent obstetric outcome. Am J Obstet Gynecol 1999;181:1216-21.

9. Meis PJ, Michielutte R, Peters TJ, Wells HB, Sands RE, Coles EC, et al. Factors associated with preterm birth in Cardiff, Wales: II. Indicated and spontaneous preterm birth. Am J Obstet Gynecol 1995;173:597-602.

10. Astolfi P, Zonta LA. Risks of preterm delivery and association with maternal age, birth order and fetal gender. Hum Reprod 1999;14:2891-4.

11. Parker JD, Schoendorf KC, Kiely JL. Associations between measures of socio-economic status and low birthweight, small for gestational age and premature delivery in the United States. Ann Epidemiol 1994;4:271-8.

12. Peacock JL, Bland JM, Anderson HR. Preterm delivery: effects of socioeconomic factors, psychological stress, smoking, alcohol and caffeine. BMJ 1995;311:531-5.

13. Goldenberg RL, Cliver SP, Mulvihill FX, Hickey CA, Hoffman HJ, Klerman LV, et al. Medical, psychosocial and behavioral risk factors do not explain the increased risk for low birth weight among black women. Am J Obstet Gynecol 1996;175:1317-24.

14. Goldenberg RL, Iams JD, Mercer BM, Meis PJ, Moawad AH, Copper RL, et al. The preterm prediction study: the value of new vs standard risk factors in predicting early and all spontaneous preterm births. Am J Public Health 1998;88:233-8.

15. Macones GA, Segel SY, Stamilio DM, Morgan MA. Prediction of delivery among women with early preterm labor by means of clinical characteristics alone. Am J Obstet Gynecol 1999;181:1414-8.

16. Meis PJ, Michielutte R, Peters TJ, Wells HB, Sands $\mathrm{RE}$, Coles EC, et al. Factors associated with preterm birth in Cardiff, Wales, I. Univariable and multivariable analysis. Am J Obstet Gynecol 1995;173:590-6.

17. Heffner LJ, Sherman CB, Speizer FE, Weiss ST. Clinical and environmental predictors of preterm labor. Obstet Gynecol 1993;81:750-7.

18. Wen SW, Goldenberg RL, Cutter GR, Hoffman HJ, Cliver SP, Davis RO, et al. Smoking, maternal age, fetal growth and gestational age at delivery. Am J Obstet Gynecol 1990;162:53-8.

19. Borges G, Lopez-Cervantes M, Medina-Mora ME, Tapia-Conyer R, Garrido F. Alcohol consumption, low birth weight, and preterm delivery in the National Addiction Survey (Mexico). Int J Addict 1993;28:355-68.

20. Spence MR, Williams R, DiGregorio GJ, McDonnell AK, Polansky M. The relationship between recent cocaine use and pregnancy outcome. Obstet Gynecol 1991;78:326-9.

21. Miller JM, Boudreaux MC. A study of antenatal cocaine use-chaos in action. Am J Obstet Gynecol 1999; 180:1427-31.

22. Wen SW, Goldenberg RL, Cutter GR, Hoffman HJ, Cliver SP. Intrauterine growth retardation and preterm delivery: prenatal risk factors in an indigent population. Am J Obstet Gynecol 1990;162:213-8.

23. Kogan MD, Alexander GR, Kotelchuck M, Nagey DA. Relation of the content of prenatal care to the risk of low birth weight: maternal reports of health behavior advice and initial prenatal care procedures. JAMA 1994;271:1340-5.

24. Hobel CJ, Ross MG, Bemis RL, Bragonier JR, Nessim S, Sandhu M, et al. The West Los Angeles Preterm Birth Prevention Project. I. Program impact on high-risk women. Am J Obstet Gynecol 1994;170:54-62.

25. Collaborative Group on Preterm Birth Prevention. Multicenter randomized, controlled trial of a preterm birth prevention program. Am J Obstet Gynecol 1993;169:352-66.

26. Main DM, Gabbe SG, Richardson D, Strong S. Can preterm deliveries be prevented? Am J Obstet Gynecol 1985;151:892-8.

27. Drazancic A. Antenatal care in developing countries. What should be done? J Perinat Med 2001;29:188-98.

28. Mercer BM, Goldenberg RL, Das A, Moawad AH, Iams JD, Meis PJ, et al. The preterm prediction study: a clinical risk assessment system. Am J Obstet Gynecol 1996;174:1885-93. 
29. Bakketeig LS, Hoffman HJ. Epidemiology of preterm birth: results from a longitudinal study of births in Norway. En: Elder MG, Hendricks CH (eds). Preterm Labor. London: Buttorworths; 1981. p. 17-46.

30. Adams MM, Elam-Evans LD, Wilson HG, Gilbertz DA. Rates and factors associated with recurrence of preterm delivery. JAMA 2000;283:1591-6.

31. Buchbinder A, Sibai BM, Caritis S, Macpherson C, Hauth J, Lindheimer MD, et al. Adverse perinatal outcomes are significantly higher in severe gestational hypertension than in mild preeclampsia. Am J Obstet Gynecol 2002;186:66-71.

32. Hnat MD, Sibai BM, Caritis S, Hauth J, Lindheimer $\mathrm{MD}$, MacPherson $\mathrm{C}$, et al. Perinatal outcome in women with recurrent preeclampsia compared with women who develop preeclampsia as nulliparas. Am J Obstet Gynecol 2002;186:422-6.
33. Sibai BM. Diagnosis and management of gestational hypertension and preeclampsia. Obstet Gynecol 2003;102:181-92.

34.Zhang J, Meikle S, Trumble A. Severe maternal morbidity associated with hypertensive disorders in pregnancy in the United States. Hypertens Pregnancy 2003;22:203-12.

35. Petrikovsky BM, Vintzileos AM. Management and outcome of multiple pregnancy of high fetal order: literature review. Obstet Gynecol Surv 1989;44:578-84.

36. Neilson JP, Verkuyl DAA, Crowther CA, Bannerman C. Preterm labor in twin pregnancies: prediction by cervical assessment. Obstet Gynecol 1988;72:719-23.

37. Martin J, Hamilton BE, Ventura SJ, Menacker F, Park MM, Sutton PD. Births: final data for 2001. Natl Vital Stat Rep 2002;51:1-102.

38. Moutquin JM. Classification and heterogeneity of preterm birth. BJOG 2003;110 Suppl 20:30-3. 